\title{
Information and Communication System for informing Users in Traffic Environment - SaforA
}

\author{
Dragan Peraković ${ }^{1}$, Marko Periša, Rosana Elizabeta Sente, Nada Bijelica, \\ Luka Brletić, Boris Bucak, Andrej Ignjatić, Vlatka Mišić, Ana Papac, Matea \\ Vuletić and Petra Zorić
}

\begin{abstract}
The aim of research is proposal of the system architecture which enables the information delivery in traffic environment to a subset of users (persons with visual impairment, hearing impairment, locomotor impairment, the elderly, children and people with no disability). The purpose of service is to provide users with accurate and real-time information and raising the level of quality of life. The proposed information and communication system architecture is based on modern technologies, while the system architecture is based on Cloud Computing for the Blind concept ensuring 24/7 support for the delivery of services and safety of users. Solution is realized in the form of smart bracelet that uses sensors to collect data from the environment and forwards them for further processing. With simulation testing and experimental methods, the work of proposed system is tested in laboratory and real-world environment which proved real-time information delivery to users. Having the ability to notify users in real time will increase the level of autonomy and safety while moving through traffic network.
\end{abstract}

\section{Introduction}

With the development of information and communication technology (ICT) comes the offer of a large number of services that are implemented in various terminal devices (TD). In order to use currently available services, market offers a

\footnotetext{
${ }^{1}$ Department of Information and Communication Traffic, Faculty of Transport and Traffic Science, University of Zagreb

Republic of Croatia

dragan.perakovic@fpz.hr
} 
wide variety of TD to users. The fact is that large number of people have a problem with adjusting to the use of functionality based on ICT. For this reason, it is necessary to develop a system which will deliver services to users in a way that they can use it separately and independently. This research proposes system architecture for informing users in traffic environment and everyday needs - Smart Assist for All (SAforA).

The system functionalities are based on the application of TD and functionalities adjusted to the needs of users are based on the conceptual system architecture.

Parents with children, the elderly, persons with disabilities and persons without disabilities make a targeted group of users (TGU) of research. The analysis of the currently available assistive technology solutions is demonstrated, their advantages and disadvantages and survey that defines the user requirements.

By applying the proposed system for informing users in traffic environment, it is expected to increase the level of quality of user's life due to possibilities to customize available information and increase their mobility.

\section{Previous research}

Currently available studies provide informations about how many people are familiar with modern technology and the possibilities that they offer. As a result, it is possible to define some of the functionalities that proposed service should provide. According to a study conducted in the United Kingdom which involved 920 people (parents with younger children), the results gave how many people are interested to use location services to know where their children are. In the United States of America, $72 \%$ of parents wants to have a real-time information about where their children are. $36 \%$ of them want their children to use a smart watch with a built-in GPS, while $39 \%$ of them would like to receive a warning from a child's smart watch if they call an emergency number. At the same time, they find a physical activity of children important, therefore, $22 \%$ of parents want to have a fitness services at children's devices to follow a number of their steps [BusinessWire 2014].

The research supported by Yeungnam University Research Grant got the results that a large number of respondents were not familiar with the concept of smart clothes. This technology may have a wide application in practice of locating children and elderly and to encourage self-reliance and independence [Park et al 2012].

There are a lot of smart bracelets that help users to monitor their health issues and their fitness capabilities like Jawbone UP3 or Garmin Vivosmart fitness bracelet. Usually they have a display that shows current information about health status of the user. Some of them have a build in applications that provide users with with information about their physical activity and gives them tips on how to improve their results. Various informations can be shown, such as: heartbeat, temperature and breathing which allows to track duration of sleeping. Some of the 
smart bracelets have built in GPS so that the users can see how many kilometers have they walked or ran. Some of the smart bracelets have the functionality to detect obstacles on the road using variety of sensors. Users get information through vibration signals. Such example is Sunu smartband that is based on Beacon technology. Another example of smart bracelet are bracelets that can locate users. They can be used to improve childrens safety. As the number of kidnapping of children increases sharply, this functionality can be considered good for trying to reduce that number. Some of the smart bracelets have the functionality to make an emergency call by pressing the button on it. In that case, parents can get an information about childrens location. Locating smart bracelets can be used for locating elderly that have dementia problems. Such example is Hemayati smart bracelet, Vega GPS bracelet etc [Zhang and Rau 2015, Huang et al 2015, Deshmukh et al 2014, Angulo et al 2015].

Previous solutions to increase the level of quality of life of individual user groups are offered in form of smart bracelet. After analysing existing solutions, it can be concluded that there are a lot of similar smart bracelets with limited functionalities and that those solutions are not suitable for all user groups. Manufactures do not think about their upgrade and thus limit the possibility of their use for other user groups.

\section{Methodology}

The main goal of this study is to propose a system architecture to deliver information services to users in traffic environment and everyday needs based on ICT. Purpose of the service is to deliver accurate and real-time information to end users. Proposal is based on research about technical characteristics of currently available sensor technologies, data transmission technologies, technologies for determining location of users and defining user requirements.

Currently available services do not satisfy needs of all user groups. Because of it, functionalities must be interoperable so that all users can use them by their own choice. The hypothesis of this study is that the application of ICT can increase the level of quality of life and improve the user's movement through transport network.

One of the goals of this study is to define service functionalities that will meet the needs of end users with purpose to increase the level of quality of life. Information about the health, social and cultural life, traffic environment and everyday needs are taken into account. For the analysis of user requirements, it is necessary to define characteristics of TGU. Persons with disabilities include those who have any restriction or lack of ability to perform certain activities which prevents their full and effective participation in society on an equal basis with others [Law on Croatian Register of Persons with Disabilities 2001]. 


\subsection{Materials and methods}

Needs of TGU were investigated in order to define user requirements and assure that the functionalities of services are fully customized to the TGU. They are related to a fully functioning in daily activities and the availability and knowledge of new solutions and services based on modern ICTs. In order to obtain relevant information, research was carried out, one in 2014 and one in 2015. The first survey was conducted in nine homes for the elderly in Zagreb.

Method of survey and interview research was carried out on the possibilities of the use of modern ICT by the elderly in order to obtain information related to their use of services based on these technologies [Black et al 2015]. From the total number of 209 respondents, 53,58\% were older than eighty years while 5,26\% were younger than sixty years

The respondents were asked about the type of their disability and 49,28\% have visual impairment, which is the highest one, while $44,98 \%$ have locomotor impairment. Figure 1 shows the reasons for the use of services based on new technologies.

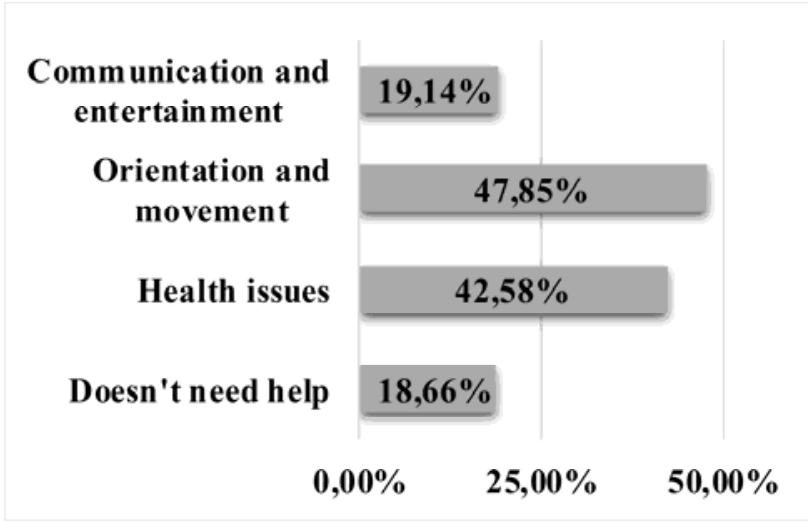

Fig. 3.1.1. Type of needed help

It was surprisingly that $73,68 \%$ respondents use MTD, where $14,19 \%$ use smartphone, while the rest of them use dumbphone. The respondents were interested $(67,94 \%)$ in the service that could help them in their everyday activities and that is customized to their needs. Figure 2 shows in what form would they use the service. 


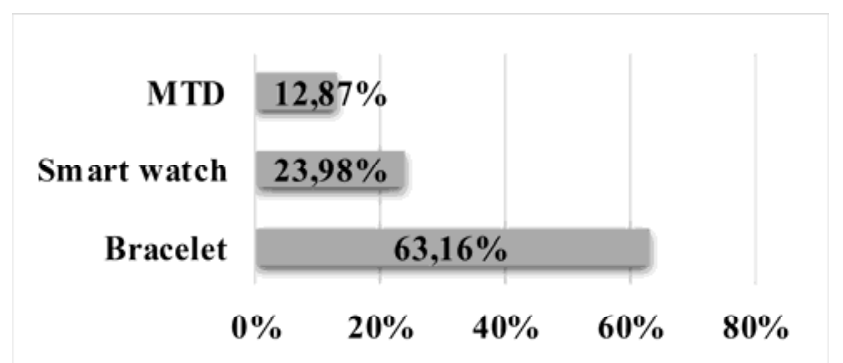

Fig. 3.1.2. Form of the service

It was shown that respondents have problems with orientation and movement so they were asked about using services that would identify them and inform them while using public transport and 53,11\% were interested in that type of service.

The second survey was conducted in collaboration with UP2DATE organization in the premises of Laboratory of Development and Research of Information and Communication Assistive Technology (ICATLab) at Faculty of Transport and Traffic Sciences in Zagreb. Research on user needs while moving traffic network was conducted by survey method. The aim of this research was to collect all relevant information about the difficulties that users face when moving through transport network and the ways and possibilities of using modern ICT in their daily activities. From total number of 112 respondents, $28,57 \%$ were in age between 21 and 24 years while 6,25\% were older than 60 years. Most of them were students, $51,79 \%$, and employees, $31,25 \%$ of them. When they were asked if they have some kind of disability, $72,32 \%$ said yes. The highest disability was vision impairment where $37,65 \%$ of respondents said that they are blind while locomotor impairment was the second one highest, $28,24 \%$. They were asked if they are satisfied with current ways of informing them about public transport, indoors, at intersections, streets and squares. In all these cases, the majority of users are not satisfied with the current way of informing. Respondents were most dissatisfied with the way of informing on at intersections, $72,73 \%$ of them. Other cases are not so far behind for such a large number of dissatisfied users and $68,18 \%$ of them stated that they were not satisfied with information on the squares.

A large number of respondents, $93,75 \%$, use MTD of which $73,33 \%$ use Android Operating System (OS). The most common use of MTD was for calls and messages but only $1,6 \%$ used them to locate objects. According to the result obtained from the survey, the two most appropriate ways to inform users are sound information using MTD (30,00\%) and visual information (27,39\%).

Due to increase the safety of users and allow them aid in dangerous situations, respondents were asked if they would like to have a service that would transmit an SOS message. Most of them replied that they would like to use this type of service $(73,64 \%)$ and would like the message to be sent to the responsible or legal person $(31,21 \%)$, emergency services $(25,48 \%)$ or spouse $(24,20 \%)$.

In the survey, there were $21,62 \%$ parents that were asked if they would use locating services to know where their children are, SOS paging if their child is in some kind of threat and detection of removal of smart bracelet. The entire TGU 
replied that they would use SOS paging service, but 90,91\% also would use other two services.

In order to determine functionalities of service to inform users in traffic, they were asked about the importance of certain information and the results are shown in Table 1. Importance of certain information that concerned information about objects, current location (CL), locating items like wallets and keys (LI), directing users with voice guidance and automatic return route (DUWV), health status (HS), open area like markets and squares (OA), barriers, automatic SOS call (SOS) and fall detection (FD) was evaluated according to their importance on a scale from 1 (very irrelevant) to 5 (very relevant).

Table 3.1.1. Importance of information for users

\begin{tabular}{l|lllll}
\multirow{2}{*}{$\begin{array}{l}\text { Object } \\
\text { CL }\end{array}$} & 1 & 2 & 3 & 4 & 5 \\
\cline { 2 - 6 } & $7,34 \%$ & $5,50 \%$ & $13,76 \%$ & $37,61 \%$ & $35,78 \%$ \\
\hline LI & $2,75 \%$ & $3,67 \%$ & $11,01 \%$ & $47,71 \%$ & $34,86 \%$ \\
\hline DUWV & $6,48 \%$ & $18,52 \%$ & $20,37 \%$ & $36,11 \%$ & $18,52 \%$ \\
\hline HS & $5,50 \%$ & $11,01 \%$ & $16,51 \%$ & $43,12 \%$ & $23,85 \%$ \\
\hline OA & $9,09 \%$ & $12,73 \%$ & $29,09 \%$ & $30,00 \%$ & $19,09 \%$ \\
\hline Barrier & $5,50 \%$ & $5,50 \%$ & $29,36 \%$ & $31,19 \%$ & $28,44 \%$ \\
\hline SOS & $2,86 \%$ & $5,71 \%$ & $15,24 \%$ & $40,00 \%$ & $36,19 \%$ \\
\hline FD & $10,09 \%$ & $10,09 \%$ & $18,35 \%$ & $27,52 \%$ & $33,94 \%$ \\
\hline
\end{tabular}

With given results, it is possible to determine the level of importance of service functionalities. Automatic transmission of SOS call 33,94\% of users consider to be very important and 36,19\% also find information about barriers very important. Navigating route with voice guidance $43,12 \%$ find important as well as information of the current location, $47,71 \%$ of them. Relevant parameters can be divided into system components and services. The parameters for the design of the proposed system are hardware and network components.

Hardware component consists of MTD and smart bracelet. Suitable ways for informing users are audio and visual informing. Network component consists of Bluetooth system, Cloud Computing for the Blind (CCfB) and Internet of Things (IoT). Bluetooth technology is used as a linking element in the system while CCfB is used for system requirements and the needs of users for real-time information. IoT is used because of the need to connect the entire system to a global network and access real-time information. 


\subsection{Service accessibility}

System has to be designed to be accessible to all users. The concept of universal design is an approach of designing products, services and the environment so that they can be usable to all people, without the need for adjustments or special design. Universal design can increase the utilization of the environment or products without significant increase in its cost while reducing the need for design changes later when capacity circumstances change [Periša et al 2014].

The proposed system for informing in the form of smart bracelet has an ergonomically friendly design. Equitable use is achieved by designing services according to customer requirements. Users will get required information in the fewest possible steps which achieves flexibility of service and low physical effort.

Content and information will be clear and easy to see or hear. Using and understanding of the entire system for all users will be easy [Mann 2005]. The service, in addition to the application of universal design, should also have designed architecture services to Ambient Assisted Living (AAL) concept [Garcia and Rodrigues 2015].

\section{Technology analysis}

With ICT it is possible to increase the level of quality of life of users if they are implemented in ways that are simple to use for all users. When designing a system, it is necessary to check the compatibility of technologies and their capabilities in order to provide real-time and accurate information.

\subsection{Bluetooth and Near Field Communication Technology}

Near Field Communication (NFC) and Bluetooth technologies are used in proposed model of assistive technology. They link users, enabling them to communicate, other transport entities and the overall traffic environment into a unified whole by applying the principles of IoT. Bluetooth technology (BLe v.4.0.) is an open standard that can be implemented in IoT and AAL concepts [Gomez et al 2012]. NFC technology is designed for use on devices that re located close to each other [Al-Ofeishat and Al Rababah 2013]. The big advantage over other technologies is that it doesn't require pairing devices which facilitates the use of technology. 


\subsection{Arduino and Raspberry Pi platforms}

For the development of a prototype smart bracelet it is possible to use Arduino platform. Arduino platform is an open-source family of microcontrollers that can be paired with various sensors. Microcontroller has modest possibilities when compared to the performances of today's computers and even MTD. Arduino UNO is small and has a low power consumptions. More advanced modules that can wirelessly connect to Arduino platform are called shields. Except them, there are a lot of programs created by Arduino community that are shared on the Internet.

With a relatively low cost price and additional modules and shields, Arduino platform is a great choice for testing and developing new concepts and ideas [14].

Raspberry Pi is also a platform that can be used as an IoT device because of its small size and possibilities of connection to the Internet and to other devices. In relation to Arduino, Raspberry Pi models have great processing power. It has an integrate Ethernet port that allows easier to connect to the Internet. Disadvantages of Raspberry Pi are high energy consumption, inability to process analog signals and interface that requires access via keyboard, mouse and computer monitor.

For those reason, Raspberry $\mathrm{Pi}$ is often used as a central processor unit that collects data from other devices and sensors and then forwards them to the $\mathrm{CC}$ for storage and processing [Periša et al 2016].

\section{Solution proposal}

Based on research done in the field of application of CC technology, it is proposed to extend the functionalities of $\mathrm{CCfB}$ concept through conceptual architecture of the system [Periša et al 2014]. The functionalities are focused on efficient delivery of information to users by using sensor technology.

As it's shown in Figure 3, system architecture consists of five elements: sensors, smart bracelet, user, MTU, terminal device (TD), mobile and web application, user database, database, influential interest groups (IIG) and functionalities of SAforA service.

Sensors located in the architecture collect data from environment of user. Data is collected in the proposed informing system, in the form of smart bracelet and are stored in the database. All data from sensor located on the smart bracelet are stored in the database. Database contains data that require further processing and adapting into information comprehensible to the end user. User database, which contains information of accounts and their settings, is a part of $\mathrm{CCfB}$ architecture of service provider. It provides necessary data for verification and adaptation of content to the user priority. Adaptation of content is performed based on user characteristics and functionalities that each user chooses.

Users can access their data through mobile applications on MTD or web applications using a web browser. These applications are integral part of CCfB archi- 
tecture (service provider part) and through these applications users demand information collected from smart bracelet. Data is processed and adapted to the needs of users and are presented as a relevant information in the application. Users can configure smart bracelet using MTD and Bluetooth connection or computer and IP/GPRC communication channel. All data from the sensors are sent to central processor unit for processing that is located in smart bracelet, where they transform into information that will be sent over the network to the database. Individual sensors located on smart bracelet can be turned on or off when setting smart bracelet. In that case, users will get only those information from sensors that are turned on.

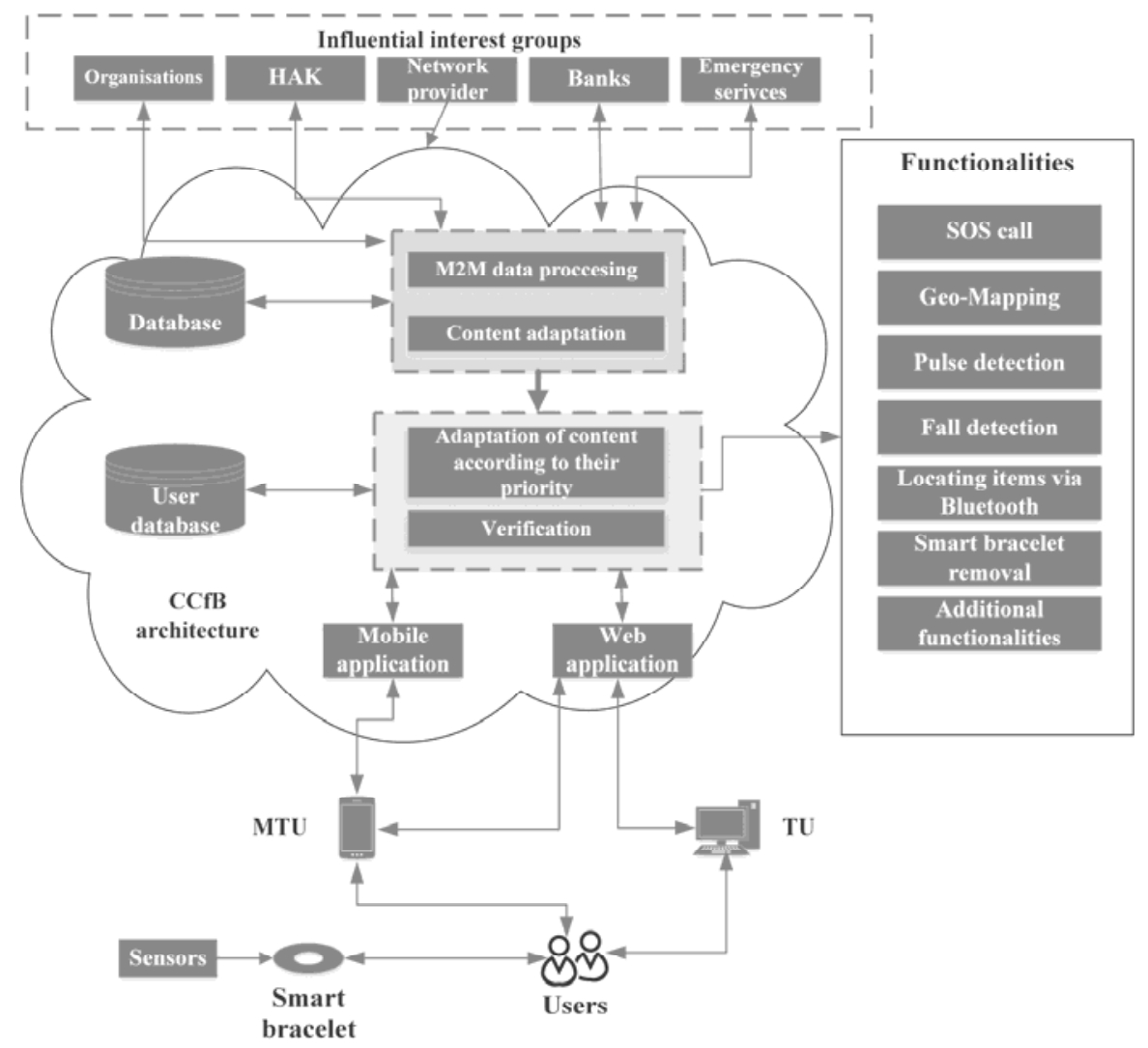

Fig. 5.1. Architecture of SAforA service

As noted above, users can configure smart bracelet in two ways: by using MTD or computer. If the smart bracelet is configured with MTD, Bluetooth connection is used. After identification and authentication, smart bracelet connects with MTD and receive configuration information that apply automatically. Configuration via computer goes through web application. User logs into his account 
and chooses settings that he wants smart bracelet to do. User accounts are stored in CCfB database of provider, who during the logging in identifies and authorize user. The user then changes the settings in smart bracelet and after saving the changes are send via IP/GPRS connectivity to smart bracelet. In case where settings were changed for more smart bracelets, settings will be send to all smart bracelets. It is possible to access web application by MTD using web browser. Application solution is able to guide the user through voice, visual and tactile information which is not possible using the web browser. For example, if user wants to get direction to some place, he needs to set a route on his MTD. After the data is processed, it's send to smart bracelet that presents them as a visual, audio or tactile information.

Important interest groups (IIG) communicate with CCfB service provider using IP protocol and available Internet infrastructure. This kind of communication is two-way and through it system can inform IIG about location and user condition, and also, they can request information about a specific user if they have permission to do so. Users can allow specific IIG to have information about them while configuring smart bracelet. This method allows IIG to have access only to that information that users allows them. Service provider is in charged for this functionality because he processes information in CCfB architecture.

\subsection{Determing the functionalities of SAforA service}

Delivery of proposed IC services based on modern ICT includes users whose needs effect on the design of the service. Stakeholders help users of the service during certain situations. Information service consists of basic and additional functionality that provide all relevant information. Use case diagram is shown in Figure 4. Additional functionalities are watch, flashlight, weather forecast/temperature, alarm reminder, fitness services, contactless payment, locking/unlocking MTD and automatic adjustment of car functions.

The functionalities are offered to all users and it is up to them to choose which functionality they want to use. There are recommendations for each user on what kind of functionalities they should use. For example, for persons with visual impairment is recommended to use fall detection functionality, Bluetooth locating items, SOS calls, route creating with an automatic return route and pulse detection.

Fall detection is a functionality that is recommended for the elderly, visually impaired persons and persons with mobility impairments who live alone and can be found in situations where they need help. After falling, user can be unconscious or can't get up and retrieve a MTD to call for help. For this purpose, a smart bracelet has to be able to detect the fall and automatically send a message to responsible persons or relevant departments. There are three cases how system that detects the fall decides how to handle received information: if the user gets up, the system will receive information through smart bracelet and won't take any emergency actions; if the user after the fall remains lying on the floor and presses the 
SOS button, the process of sending an emergency message will start; it he user after the fall remains unconscious, there is a time frame of few seconds in which the system should detect if the user got up. By using accelerometer and gyroscope, smart bracelet can determine whether the user stood up and, if not, it will send the SOS message to the predetermined destination.

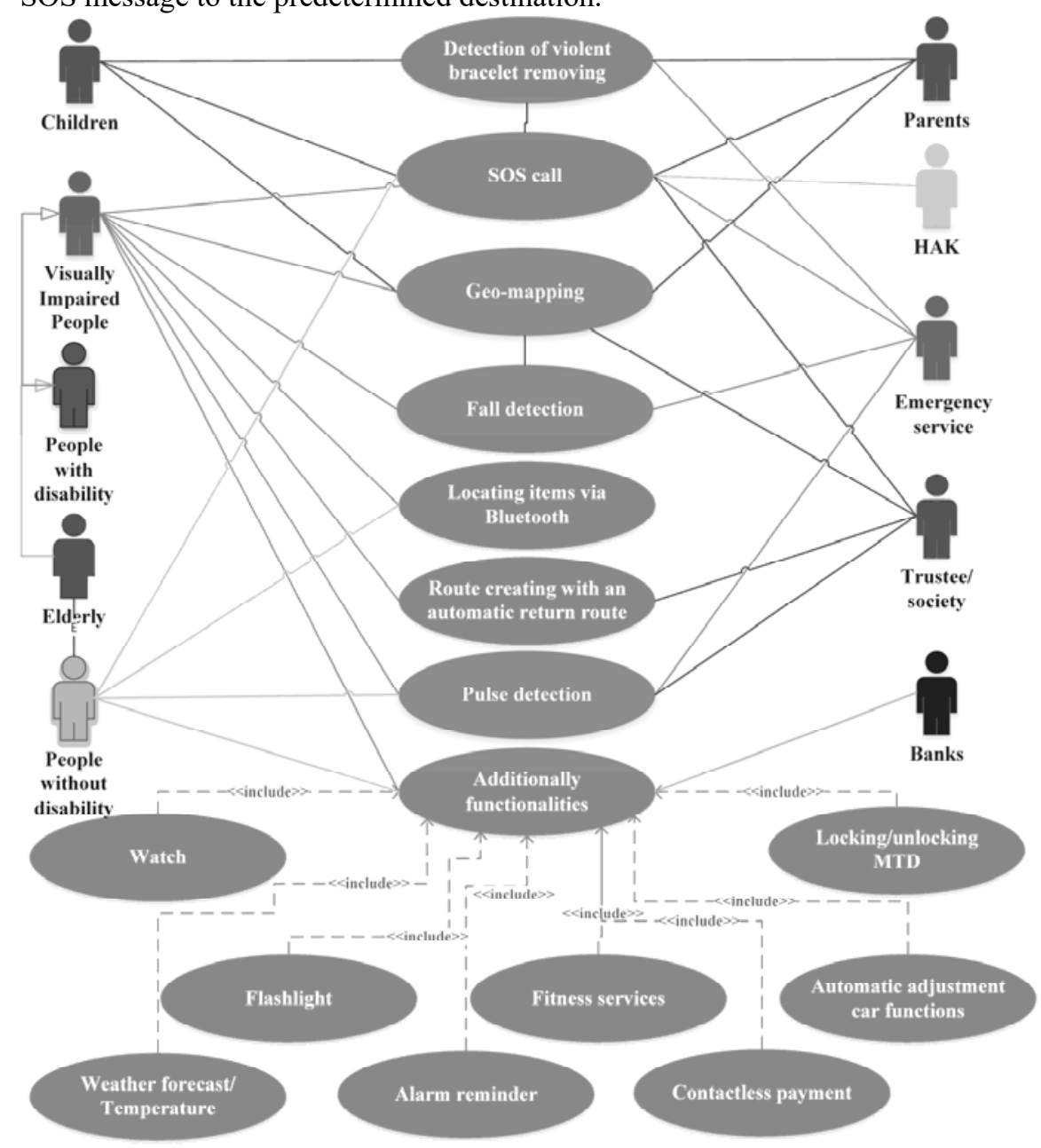

Fig. 5.1.1. Basic and additional functionalities

In case of violent removal of smart bracelet, user is not able to press the SOS button. In such situation, it is necessary to automatically send the information that the smart bracelet is removed violently. With the violent removal of the smart bracelet, a thin wire that circles the smart bracelet is broken and the distress message is sent to responsible persons. The message is automatic and includes the location and time of the event. To be able to see the difference between normal and 
violent removal of the device, there is a button that must be pressed when the device wants to be removed normally. By pressing and holding the button, distress message won't be sent. To increase the safety of the users, there is a time period within there must be no removal of the device even if you press the security button. This reduces the risk of unauthorized removal of the smart bracelet.

GPS module can be used to define the range of movement and locate the user. Studies conducted in ICATLab (The Laboratory of Development and Research of Information and Communication Assistive Technology) tested the reliability of GPS application solutions on MTD and the accuracy interval ranged from 1 to 40 [m]. Different kind of MTD were used: Sony Xperia Z, Z1 and Z3, Samsung Galaxy S4 and S6 and Nokia Lumia 925. For the children and elderly that have caregivers, it is possible to set the geographic area within they can move. In case of leaving the area within a set period of time, the responsible person or association will receive alerts and take further actions. User can also choose via the mobile application a destination after which he will get the information about the fastest route to destination. The route will be selected based on the characteristic of impairment that user has.

For example, if the user has visual impairment, presented route will be the one that has Beacon devices set on intersections. This allows better guiding and locating the user because user is guided by tactile information which he gets through vibrating motors on the smart bracelet and/or through audio signal that produces the smart bracelet and is activated only in the presence of the Beacon device that is set on the intersection. In addition to external guidance, it is possible to use the functionality of the inner guidance that also uses Bluetooth Beacon technology. Pulse monitoring can be interesting to a wider range of users regardless of whether they are impaired persons, the elderly or people with health problems. Users can track their pulse for medical purposes or simply to see how sports affects the heart. The functionality is implemented by using modules for heart measuring rate. The sensor is placed on the smart bracelet that processes the received data and afterwards sends them to a computer cloud where the information are being stored.

\subsection{Proposal for the development of the smart bracelet}

For the development of smart bracelet, it is proposed to use Arduino platform. In order to determine whether it is possible to collect the necessary data from the sensors and by their reading provide defined functionalities of service, experimental methods were conducted in ICATLab. The smart bracelet needs to be designed in a way so that it can be used by all user groups. The proposal of the smart bracelet is given in Figure 5. The modules are located on the smart bracelet in a way to reduce the danger to the users and allows them to use all the functionalities according to their needs. Smart bracelet can be used with a minimum effort and situations as accidentally key pressing are avoided. 


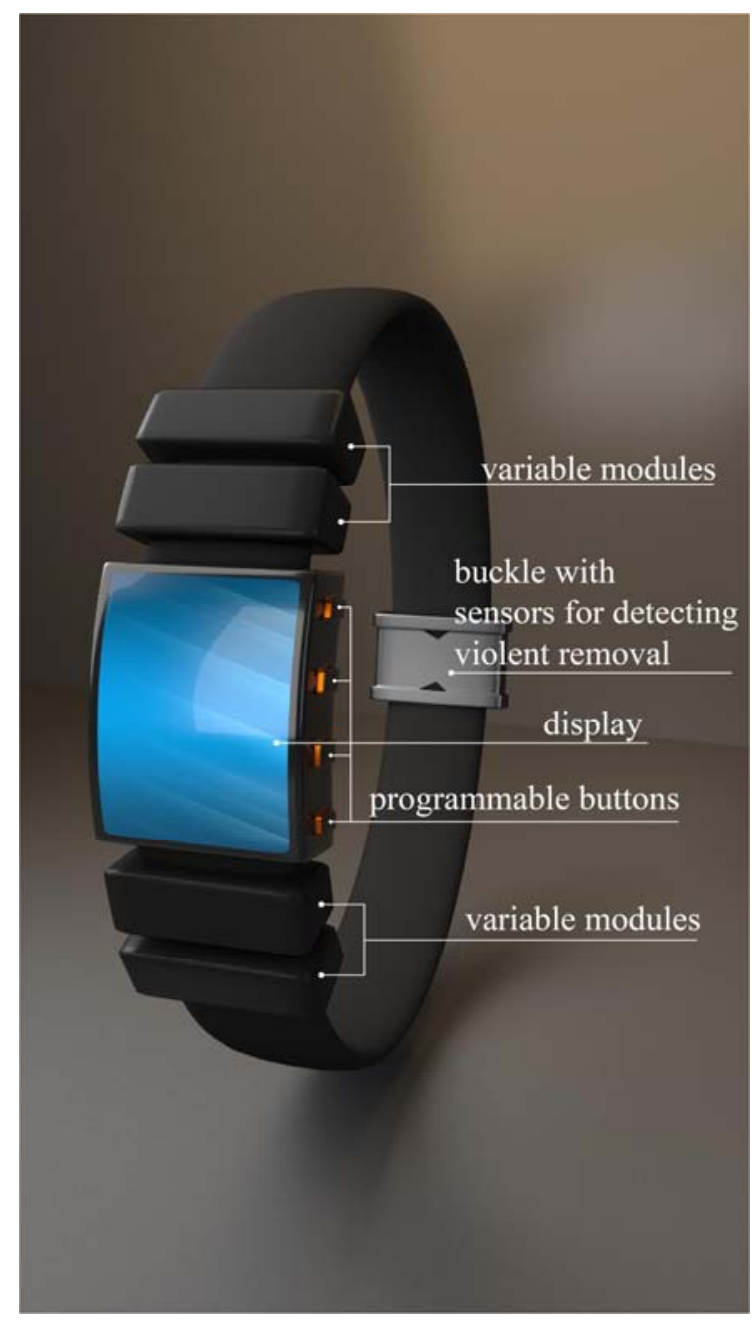

Fig. 5.2.1 Conceptual overview of the smart bracelet

In order to properly calibrate the acceleration and vibration sensors, test have been conducted in a laboratory environment. Arduino UNO was connected with a module for measuring acceleration and vibration detection module by using USB cable that is connected to a laptop. By this, the whole platform becomes mobile and it is possible to conduct tests of fall detecting. The procedure was repeated until they got the approximate sensor value that reduces the number of falsepositive results.

Raspberry Pi platform was used to simulate CCfB because of his ability to simulate a large computer network. Testing was conducted on the Raspberry Pi Model $\mathrm{B}$ (RPi3), a miniature computer with low energy consumption that uses operating system based on the Linux kernel [Jain et al 2014]. It has the ability to use Ncat 
program that can send and receive TCP and UDP packages on open ports. The command syntax is simple and is able to redirect packages to other ports even when the received packed is in the different protocol than it was during the sending. The system which sends out an SOS message consists of assistive device, communication channel, MTD, servers and sensors. Assistive device is based on the Arduino platform, communication channel is based on IP protocol and in laboratory environment it represents a local network while in real environment represents GSM/GPRS, MTD receives a text message and CCfB represents a server. $\mathrm{RPi} 3$ is used to represent mentioned server, runs a Ncat program and waits on receiving packages from the network. The sensors are located on the assistive device and all readings are processed on the Arduino platform.

The first scenario is that the person presses the SOS button on the assistive device. By pressing the SOS button, data packet is sent via GSM/GPRS mobile network to a server that is located in the CCfB. According to the user needs, a close person can be defined as a person that will receive an SOS message. That part of communication is simulated by SMS to a given number of MTD. If the message was sent, the IC system will receive an information. In case that the information wasn't set, the system will forward the message to emergency services. Message forwarding is tested by using the e-mail service and can be send automatically by using RPi3 [Jain et al 2014].

In the second scenario the example of fall detection is tested to see if the SOS message will be send. Sensors on Arduino UNO platform detect vibrations and acceleration towards the Earths surface. Arduino UNO, based on the program that is installed on it, recognizes whether the person is going down the stairs or moving in the elevator or if the person has fallen. When collecting data from the sensor detects a fall, SOS packet is sent using Wi-FI module and local IP network to RPi3. After receiving the packet, system is being informed that the user has fallen and that within a defined set of time did not get up. The simulation in the laboratory stops at this step.

\section{Conclusion}

Different characteristics, interests, skills and the level of disability makes users different from each other. Universal design, as an approach in designing products, service and environment, allows the integration of user characteristics in one system without the need for adaption. Currently implemented solutions on the market don't provide the possibility of equal use of experience to all consumers.

Data about daily needs of users was collected by using the methods of surveying and interviewing. According to the results of questionnaires relevant parameters were defined. These parameters were used in the design of the system intended for informing users in traffic environment. Research results also gave the information about what type of functionalities should system provide and they were divided into basic and additional functionalities. On the basis of the study and the experimental method it has been established that the optimal solution to 
customer needs comes in a form of smart bracelet in combination with an application to MTD.

Conceptual architecture of the system for informing users - SaforA is proposed on the basis of defined functionalities. Given the currently available solutions on the market and existing modern technology, the proposed architecture is IC system based on the $\mathrm{CCfB}$ concept. The above architecture is currently one of the better choices for connecting accurate and real-time information in one common system that is available to the user. System with multiple stakeholders involved in the formation of the information available to the users is based on the mentioned architecture.

Real-time communication between user and the system was simulated using experimental method with the use of laboratory equipment in laboratory and realworld environment. After conducted simulation it was proved that it is possible to make the data transfer from a terminal deice to the system that is based on the CCfB concept. Safety of user information and the $24 / 7$ delivery is provided. The hypothesis that using ICT can increase the level of quality of life and improve the user's movement through the transport network was proved through conducted researches and simulation verification. By providing accurate and real-time information it is possible to increase autonomy and independence of the user while moving through a part of the transport network.

In future research, it is proposed to explore the possibilities of new technologies in order to design new module for expanding and adjusting the service. Implementation of new modules that add new functionalities within the service is simple because of the modularity of the proposed bracelet.

Acknowledgments This research has been carried out as part of the project "System of automatic identification and informing of mobile entities in the traffic environment", Faculty of Transport and Traffic Sciences, University of Zagreb, 2016. The research was awarded with Rector's award at University of Zagreb, Zagreb in 2016.

\section{References}

Al-Ofeishat, H. and Al Rababah, M. (2012) Near Field Communication. International Journal of Computer Science and Network Security 12(2):93-99

Angulo, I., Onieva, E., Perallos, A., Salaberria, I., Bahillo, A., Azpilicueta, L., Falcone, F., Astrain, J.J and Villadangos, J. (2015) Low Cost Real Time Location System Based in Radio Frequency Identification for the Provision of Social and Safety Services. Wireless Personal Communications 84(4): 2797-2814. doi: 10.1007/s11277-015-2767-6

Black, R. D., Weinberg, L. A. and Brodwin M.G (2015) Universal Design for Learning and Instruction: Perspectives of Students with Disabilities in Higher Education. Exceptionality Education International 25(2): 1-26

BusinessWire (2014) Parents Want Technology to Track Their Children. http://www.businesswire.com/news/home/20141111005176/en/Parents-Technology-TrackChildren 
Deshmukh, A., Mishra, A.K., Patil, V. and Saraf K. (2014) Wireless Personal Safety Bracelet. International Journal of Computer Appliations 107(23): 11-13

Garcia, N. M and Rodrigues, J. J. (2015) Ambient Assisted Living, USA

Gomez, C., Oller, J. and Paradells, J. (2012) Overview and Evaluation of Bluetooth Low Energy: An Emerging Low-Power Wireless Technology. Sensors 12(9):11734-11753

Huang, J.C.S., Lin Y.T., Yu, J.K.L., Liu, K. and Kuo, Y.H (2015) A Wearable NFC Wristband to locate Dementia Patients through a Participatory Sensing System. In: International Conferenece on Healthcare Informatics, Dallas, 21-23 October 2015

Jain, S., Vaibhav, A. and Goyal, L. (2014) Raspberry Pi based Interactive Home Automation System through E-mail. In: 2014 International Conference on Optimization, Reliability, and Information Technology - ICROIT, Rachna International University, Manav, 6-8 February 2014.

Law on Croatian Register of Persons with Disabilities, NN 64/01, Zagreb: Narodne novine

Mann, W. C. (2005) Smart Technology for Aging. Disability and Independence, Canada

Park, S., Harden, A. J., Nam, J., Saiki, D., Hall, S. S., Kandiah, J. (2012) Attitudes and Acceptability of Smart Wear Technology: Qualitative Analysis from the Perspective of Caregivers. International Journal of Human Ecology, 13(2): 87-100

Periša, M., Jovović, I. and Peraković, D. (2014) Recommendations for the Development of Information and Communication Services for Increasing Mobility of Visually Impaired Persons. In Proceedings of the Conference Universal Learning Design, Masaryk University, 9-11 July 2014

Periša, M., Peraković, D. and Šarić, S. (2014) Conceptual Model of Providing Traffic Navigation Services to Visually Impaired Persons. Promet - Traffic \& Transportation 26(3): 209-218. doi: $10.7307 /$ ptt.v26i3.1492

Periša, M., Sente, R.E. and Brletić L. (2016) Proposal of Information Communication Technology Architecture for People with Disability. In: The $4^{\text {th }}$ Online Scientific Conference - ScieConf, 6-10 June 2016

Zhang, Y. and Rau, P.L.P. (2015) Playing with multiple wearable devices: Exploring the influence of display, motion and gender. Computers in Human Beahviour 50: 148-158 